\title{
On-chip NIR optical spectrometer based on polymeric waveguide and metallic nano-structures
}

\author{
Maurine Malak*a $^{*}$, Konstantins Jefimovs ${ }^{\mathrm{b}}$, Irene Philipoussis ${ }^{\mathrm{a}}$, Edoardo Alberti ${ }^{\mathrm{c}}$, Toralf Scharf ${ }^{\mathrm{a}}$ \\ ${ }^{a}$ Ecole Polytechnique Fédérale de Lausanne, Institut de Microtechnique, Optics and Photonics \\ Technoloy laboratory, Rue de la Maladière 71b, CH-2000 Neuchâtel, Switzerland; \\ ${ }^{\mathrm{b}}$ Recently with Paul Scherrer Institut, 5232 Villigen PSI, Switzerland; \\ ${ }^{\mathrm{c}}$ Micos Engineering GmbH, Überlandstrasse 129, CH-8600 Dübendorf, Switzerland
}

\begin{abstract}
In this work, we report about optical spectrometry using gold nano-structures printed on a polymer based integrated optical waveguide. The optical waveguide is a single mode buried waveguide, having dimensions of $3 \times 2.2 \mu \mathrm{m}^{2}$. It is made from a combination of photo-polymerizable materials and is fabricated by photolithography on a glass substrate. To sense the electric field inside the waveguide, a gold nano-coupler array of thin lines ( $50 \mathrm{~nm}$ thick and $8 \mu \mathrm{m}$ length) is embedded on top of the aforementioned waveguide. They are produced by E-beam lithography. The array pitch is 2.872 $\mu \mathrm{m}$ and the number of lines 564, which yields an array of $1.619 \mathrm{~mm}$ length. The device is enclosed with a glass superstrate to prevent it from dust and destruction. Both waveguide ports are polished and the output port in particular, is coated with a thin gold layer to assimilate a mirror and hence, it enables the creation of stationary waves inside the structure.

The measurement procedure involves light injection using a single mode fiber carrying both visible light (658nm) and infrared light $(785 \mathrm{~nm})$, used for alignment and measurement purposes respectively. Stationary waves generated inside the guide constitute the spatial interferogram. Locally, light is out-coupled using the nano-couplers and allows measuring the interferogram structure. The resulting pattern is imaged by a vision system involving an optical microscope with a digital camera mounted on-top of it. Signal processing, mainly based on Fast Fourier transform is performed on the captured signal to extract the spectral content of the measured signal.
\end{abstract}

Keywords: Polymer waveguide, Epocore/Epoclad, nano-antennas, nano-couplers, field samplers, spectrometer

\section{INTRODUCTION}

Since its early beginning in $1666^{1}$, spectroscopy acquired a large interest thanks to their numerous applications ${ }^{2}$. The atomic $^{3}$ and molecular ${ }^{4,5}$ structures are identified by their absorption or emission spectra and there comes the importance of spectroscopy to the different fundamental sciences: Chemistry, Physics and life sciences. Therefore, a complete comprehensive understanding of quantum mechanics ${ }^{3}$, blackbody radiation theory, atomic structure and spectra could not be achieved without advancement of spectroscopic studies. Spectroscopic techniques can be classified according to the type of radiative energy involved in the interaction (electromagnetic waves, mechanical waves or radiation pressure waves). They can also be classified according to the nature of the light-matter interaction occurring during the analysis (absorption, emission, reflection...). Spectroscopy can be achieved using several techniques ${ }^{6}$ : Cavity ring down spectroscopy $^{7}$, cavity enhanced absorption spectroscopy ${ }^{8}$, Raman spectroscopy ${ }^{9}$, time resolved spectroscopy, mass spectroscopy $^{10}, \mathrm{UV}$-visible spectroscopy, nuclear magnetic resonance ${ }^{11}$, and Fourier Transform spectroscopy ${ }^{12}$ (FTS) as presented in this research work.

In order to fulfill the increasing demand for spectral analysis, various optical components and Microsystems have been conceived. Amongst, we can cite: Fabry-Perot ${ }^{13}$ and Michelson interferometers ${ }^{14,15}$, Array Waveguide Grating ${ }^{16}$ and Standing wave Fourier Transform spectrometer ${ }^{17}$. Recently, a study aiming miniaturization has been conducted on Focal Plane Array Spectrometers ${ }^{18}$. Herein, we report about the realization of a compact, fully integrated FTS based on micronano structures, combining a micron sized optical waveguide with an array of nano-samplers.

*maurine.malak@epfl.ch; phone +41 216954 463; fax +41 216954 201; opt.epfl.ch

MOEMS and Miniaturized Systems XIII, edited by Wibool Piyawattanametha,

Yong-Hwa Park, Proc. of SPIE Vol. 8977, 89770S · (c) 2014 SPIE

CCC code: $0277-786 X / 14 / \$ 18 \cdot$ doi: $10.1117 / 12.2037218$

Proc. of SPIE Vol. 8977 89770S-1 


\section{FABRICATION AND DESIGN}

\subsection{Waveguide fabrication process}

Epo-materials (provided by Micro resist technology $\mathrm{GmbH}$ ) are used to produce the optical waveguides. Epocore/Epoclad materials were chosen to realize the optical device because they offer unique features compared to other polymeric materials. Among others, they are characterized by high transmittance in the near IR range and the possibility of tuning the refractive index. The fabrication process shown in Figure 1 is deployed for the device realization, it yields symmetric buried waveguides. As noticed from the schematic, waveguides fabrication is based mainly on photolithography and thin film deposition. For more details about the process steps and parameters, the interested reader can refer to ${ }^{19}$. Details about nano-antennas fabrication and optimization are described in the upcoming sections. It is worth to mention that an optional black layer can be deposited over the last Epoclad layer. This optional layer will serve as a light absorber that suppresses parasitic stray light as dictated by the spectrometry application.

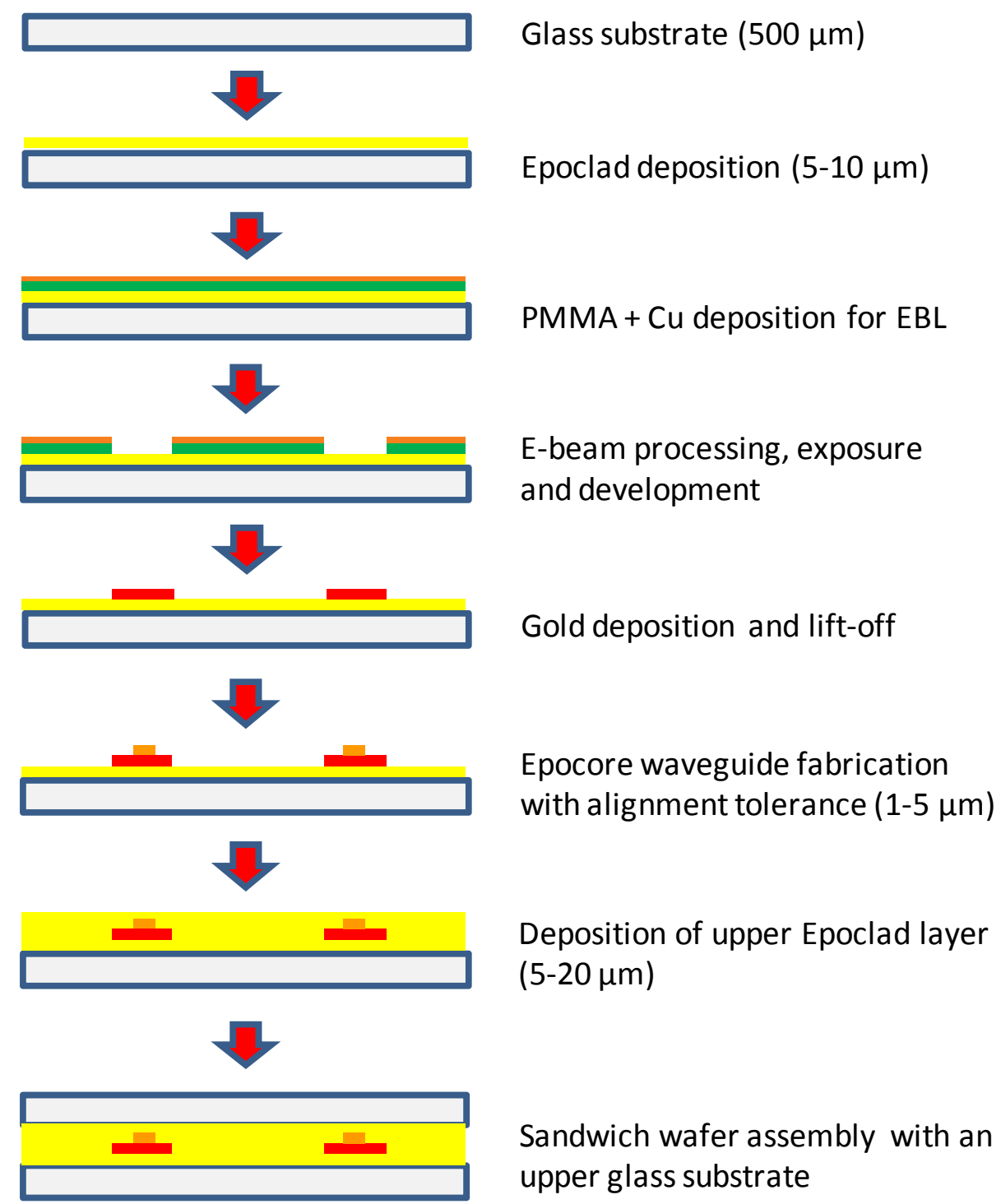

Figure 1. Schematic illustration of the fabrication process for the sandwich wafer assembly. The drawings highlight the fabrication of the waveguides, the underneath nano-antennas and the final packaging with the top glass superstrate. 


\subsection{Nano-antennas fabrication process}

Antenna structures were fabricated by a standard lift-off technique (schematized in Figure 2 below) over Epoclad layer deposited on top of fused silica wafers. In fact early attempts to manufacture them on the baseline waveguides were unsuccessful mainly because it is difficult to manage nanostructure deposition processes on prestructured surfaces of several micrometer height. The finally established nano-antennas fabrication process can be described as follows: An Epoclad layer is laid on top of a typical fused silica wafer used as a substrate. Then a PMMA layer (used as a resist) is deposited on top of the Epoclad. Since fused silica is an insulator, a copper $(\mathrm{Cu})$ conductive layer was added on top of the resist in order to avoid charging during the electron beam lithography (EBL) step. After EBL exposure, the Cu layer is removed in weak nitric acid and PMMA is developed in MIBK:IPA solution. After the development, a short oxygen plasma step is applied to clean possible organic residuals in the developed areas of PMMA. Then, the antenna material (typically, gold) is evaporated and then actual lift-off in acetone is performed. So the metal deposited on top of resist is removed together with the dissolved resist, while the metal deposited directly onto the substrate surface in the developed areas stays. We can deduce that in order to achieve a highest resolution, reproducibility and quality, the exposure and resist development parameters should be properly adjusted. Also, optimization of the antenna fabrication process is required at different stages of the sample fabrication and the most important step is the EBL.

\section{Substrate and layers}

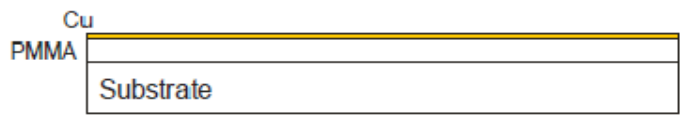

2. EBL, Cu removal and resist development

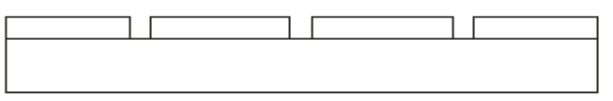

\section{Metal deposition}

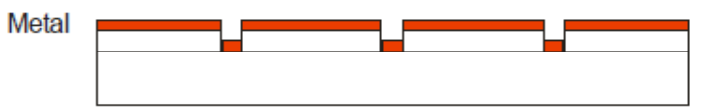

4. Lift-off

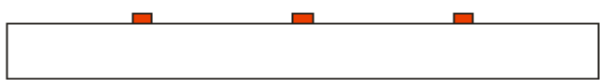

Figure 2. Schematics of antenna fabrication by electron beam lithography (EBL) and lift-off.

\subsection{Optimization of the antenna fabrication parameters}

Our original idea was to limit the EBL energy to $5 \mathrm{keV}$ in order to avoid the so-called proximity effect - that is: exposure of the neighboring area due to scattering of electrons in the substrate. The proximity effect is known to cause the neighboring line broadening and even shifting. However, it is noticed that the minimum linewidth that we could get with $5 \mathrm{keV}$ energy is around $55 \mathrm{~nm}$. This is due to scattering of low energy electrons in the copper conductive layer deposited on top of PMMA resist. While the resolution level is still acceptable, another problem is encountered which is the lack of quality and reproducibility (Figure 3). Therefore, we decided to switch to $30 \mathrm{keV}$ energy. Figure 4 shows examples of plasmonic nano-antennas of different length fabricated using $30 \mathrm{keV}$. Note that antennas of different length have essentially the same linewidth, which means that the proximity effect is negligible. It is found that the structures are very insensitive to dose variations, hence a change of dose by a factor of two results in a linewidth change of $20 \mathrm{~nm}$ (from 30 to $50 \mathrm{~nm}$ ). Thus, an energy of $30 \mathrm{keV}$ is used as an optimal value for all the future fabrication. 


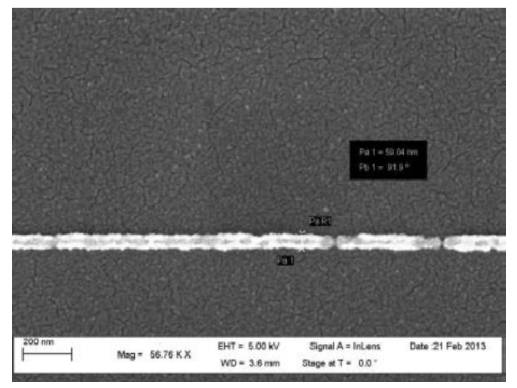

Figure 3. Examples of antenna fabricated using $5 \mathrm{keV}$ energy. Irregularities are observed in the deposited nano-antennas.

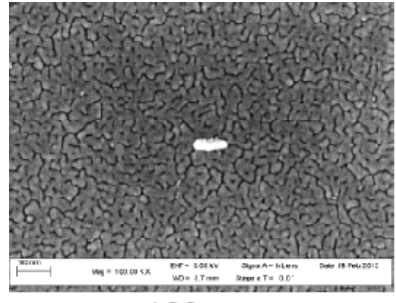

$100 \mathrm{~nm}$

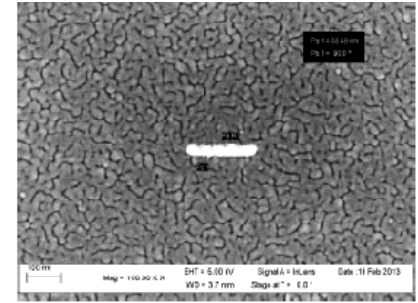

$200 \mathrm{~nm}$

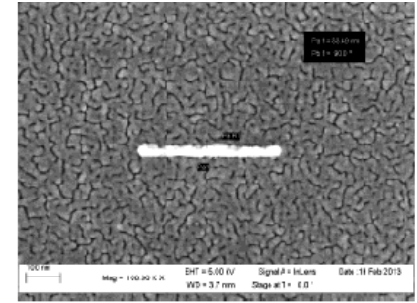

$400 \mathrm{~nm}$

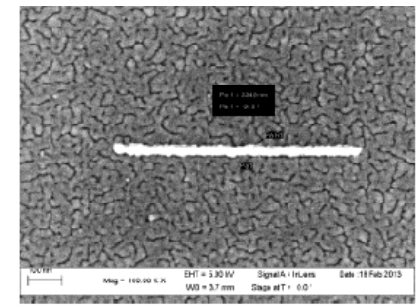

$700 \mathrm{~nm}$

Figure 4. Examples of antenna fabricated using $30 \mathrm{keV}$ energy. Higher reproducibility, less defects and higher tolerance to dose is observed, compared to $5 \mathrm{keV}$.

\subsection{Device design}

The device layout shown in Figure 5 consists of a micron-sized buried, rectangular optical waveguide on top of which gold nano-antennas acting as field samplers are embedded. The waveguide is realized by photolithography in Epoxy material (Epoclad/Epocore). The core/clad refractive index difference $\Delta \mathrm{n}=8.2 \times 10^{-2}$ leads to typical waveguide dimensions of $3 \times 2.2 \mu \mathrm{m}^{2}$, chosen to assure single mode operation at a wavelength of $784 \mathrm{~nm}$. Nano-antennas, produced by EBL, have dimensions of $50 \mathrm{~nm} \times 20 \mathrm{~nm} \times 8 \mu \mathrm{m}$. They are arranged in a linear array whose period is $2.87 \mu \mathrm{m}$ and overall length is $1.619 \mathrm{~mm}$. The overall device is sandwiched between two glass substrates to preserve it from the environmental conditions. Another step involves polishing the input/output edges of the chip to provide very smooth interfaces needed for light injection at the device input and for the mirror formation at the output of the device. Finally, a thin gold layer is evaporated on the output interface to integrate the mirror to complete the spectrometer elements.

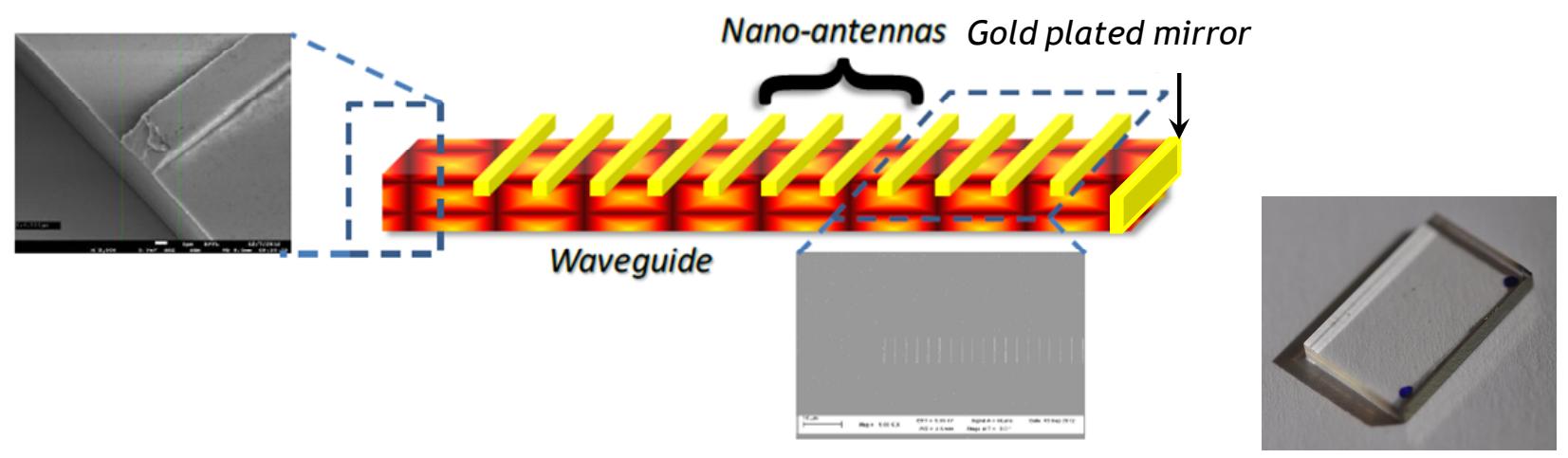

Figure 5. (Left) Schematic illustration of the device wit SEM photos showing the waveguide and the nano-antennas. (Right) Chip with a gold mirror on one side. The side where the gold mirror is located is indicated by the blue points. 


\section{OPERATION PRINCIPLE AND ANALYTICAL MODELING}

The operation principle of the system can be described as follows: Light injected into the optical waveguide in the forward direction, is back-reflected by the mirror and leads to formation of a standing wave pattern; hence an interferogram is created inside the guide. Here comes the role of the nano-antennas which act as local field sensors that out-couple a power level corresponding to the spatial light distribution of the interferogram created underneath. The sampled data are captured from the top by a microscope equipped with a camera and high resolution optics. Then, the data are treated to extract the spectral characteristics of the signal and Fourier transform is used to deduce the different spectral components propagating inside the device.

In order to understand the outcome of the Fourier Transform result, a simplified analytical model was devised to study the behavior of the lightwave propagating inside the device.

A wave propagating in the forward direction is given by:

$$
E_{f w d}=E_{0} e^{j \beta z}
$$

Where $E_{0}$ is the electric field amplitude and $\beta$ is the wave propagation constant, given by, $\beta=\frac{2 \pi}{\lambda} n_{\text {eff }}, n_{\text {eff }}$ being the effective refractive index of the mode propagating inside the guide.

Similarly, a wave propagating in the backward direction is given by:

$$
E_{b w d}=E_{0} e^{-j \beta z}
$$

Then, the total field amplitude of the standing wave is given by the sum of the forward and backward waves:

$$
E_{t o t}=E_{f w d}+E_{b w d}=E_{0} e^{j \beta z}+e^{j \pi} \cdot E_{0} e^{-j \beta z}
$$

Where, the phase $\pi$ accounts for the additional phase acquired upon reflection from the mirror.

After mathematical manipulation, we obtain:

$$
\left|E_{t o t}\right|^{2}=2\left|E_{0}\right|^{2}\{1-2 \cos (2 \beta z)\}
$$

The previous equation pertains to the envelope of the standing wave pattern. Hence, the spectral content of the sampled signal involves, among others, Fourier components of the injected wavelength $\lambda$ scaled down by $2 n_{\text {eff. }}$. It is worth to mention that the nano-samplers have a noticeable impact on the signal spectrum. In fact, the nano-antennas introduce, in addition, to the components of interest, several side peaks in the spectrum. However, the full analytical model involving the nano-antennas is out-of-scope with respect to this specific case of study and will not be reported herein.

To render this model simple and comprehensive, some basic assumptions have been considered:

1. The mirror is ideal and free from roughness and hence, the wave amplitude is kept unaltered before and after reflection. This assumption reinforces the condition of a scattering free interface with a reflection phase of $\pi$.

2. The attenuation in the guide is negligible.

\section{CHARACTERIZATION AND MEASUREMENTS}

\subsection{Optical setup}

The deployed optical setup is illustrated in Figure 6. Both visible $(658 \mathrm{~nm})$ and infrared light $(784 \mathrm{~nm})$ are used for alignment and measurement purposes, respectively. The infrared light is connected to an optical isolator to suppress any power fed back to the source and then, it assures stability of the emitted laser with a clean mode profile. The visible light, instead, is connected to an optical attenuator to enable intensity control. The isolator output and the attenuator output are combined by a directional coupler. The coupler output is in turn, connected to a polarization splitter to divide the polarizations into TE/TM obtained at each output port of the splitter. Finally, a polarization maintaining fiber (PMF) is used at the far end of the setup to transmit only one of the splitted polarizations (TE or TM) to the input of the tested device. The PMF is aligned using Newport 5-axis micro-positioners and the device is aligned using Newport 2-axis translational micro-positioners. 
The standing wave pattern generated inside the device is imaged using an optical system involving high resolution objective lenses having magnifications of 20X and 100X (provided by Mitutoyo), a Nikon microscope and a CMOS camera (provided by Mightex). The captured photo is treated in Matlab to extract the spectrum of the injected signal.

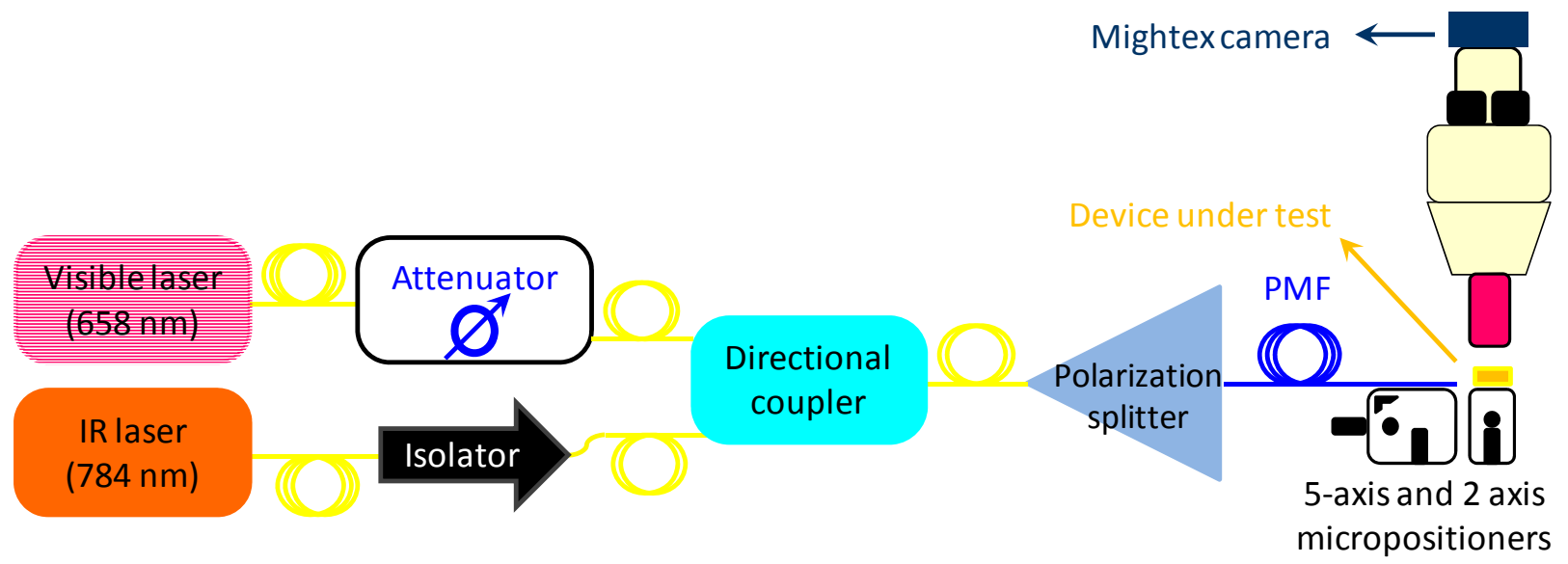

Figure 6. Schematic representation illustrating the arrangement of the optical components of the setup used to characterize the micro-spectrometer

\subsection{Characterization and measurements}

TE polarization injected into the waveguide generates a stationary wave interference pattern. Measurements carried with visible light at $658 \mathrm{~nm}$ and Mitutoyo microscopic objectives (5X, 20X and 100X) were used together with Mightex camera to capture standing wave pattern for later analysis with Fast Fourier Transform (FFT) in Matlab (Figure 7). Analysis reveals different spectral components according to the deployed objective lens.

With the 100X objective, we can identify the fundamental component and the harmonics of the nano-antennas spaced by intervals of $2.87 \mu \mathrm{m}$. Moreover, we can identify two side peaks located at $157.6 \mathrm{~nm}$, close to the DC peak, they pertain to the calibrated pixeling effect of the camera. On another hand, with the 20X objective, we can identify other peaks in addition to the nano-antennas component located at $2.887 \mu \mathrm{m}$ and the component of the calibrated pixels located at 0.8 $\mu \mathrm{m}$. Those extra peaks arise from the convolution between the aforementioned peaks at $(2.887 \mu \mathrm{m}$ and $0.8 \mu \mathrm{m})$ and they are located at $2.1 \mu \mathrm{m}$ and $4.2 \mu \mathrm{m}$. For the $5 \mathrm{X}$ objective, we observe two sets of side peaks. The set located at $232 \mathrm{~nm}$ is mainly due to the laser wavelength $(658 \mathrm{~nm})$ but scaled down by $2 n_{\text {eff }}$ as predicted by the analytical model.

From this analysis, we can say that the injected light can be deduced only when the $5 \mathrm{X}$ objective is used for conducting the measurements because a large field of view is covered with the $5 \mathrm{X}$ objective and the captured interferogram involves more wavelength periodicity than with the $20 \mathrm{X}$ or the $100 \mathrm{X}$ objectives. Hence, the source spectral component can be retrieved easier with the $5 \mathrm{X}$ objective. It is worth to mention that other peaks are also present in the FFT curves (in the range of 30-60 nm) but the reason of their presence is not yet known. In addition, measurements have been conducted at $783 \mathrm{~nm}$ and the device exhibits similar behavior to that observed at $658 \mathrm{~nm}$.

\subsection{Discussion}

The presented results need careful reconsideration since the measured spectra are heavily undersampled compared to classical FTS where one uses oversampling to recover the measured spectrum in great detail. As noticed, the observation field reduces as the objective lens magnification increases and hence, the number of nano-antennas available for sampling reduces. This limited number of nano-antennas leads to a limited resolution and the finite distance between the nanoantennas to undersampling. In addition, the different lens magnifications $(5 \mathrm{X}, 20 \mathrm{X}, 100 \mathrm{X})$ create different spectral resolution (gradually degrading for high magnification) and different spectral ranges (gradually increasing for high magnification). The FFT range being related to the camera pixel size, number of pixels, and the lens magnification. On another hand, the resolution is related to the previous set of parameters in addition to the effective number of nanoantennas captured by the field of view. 

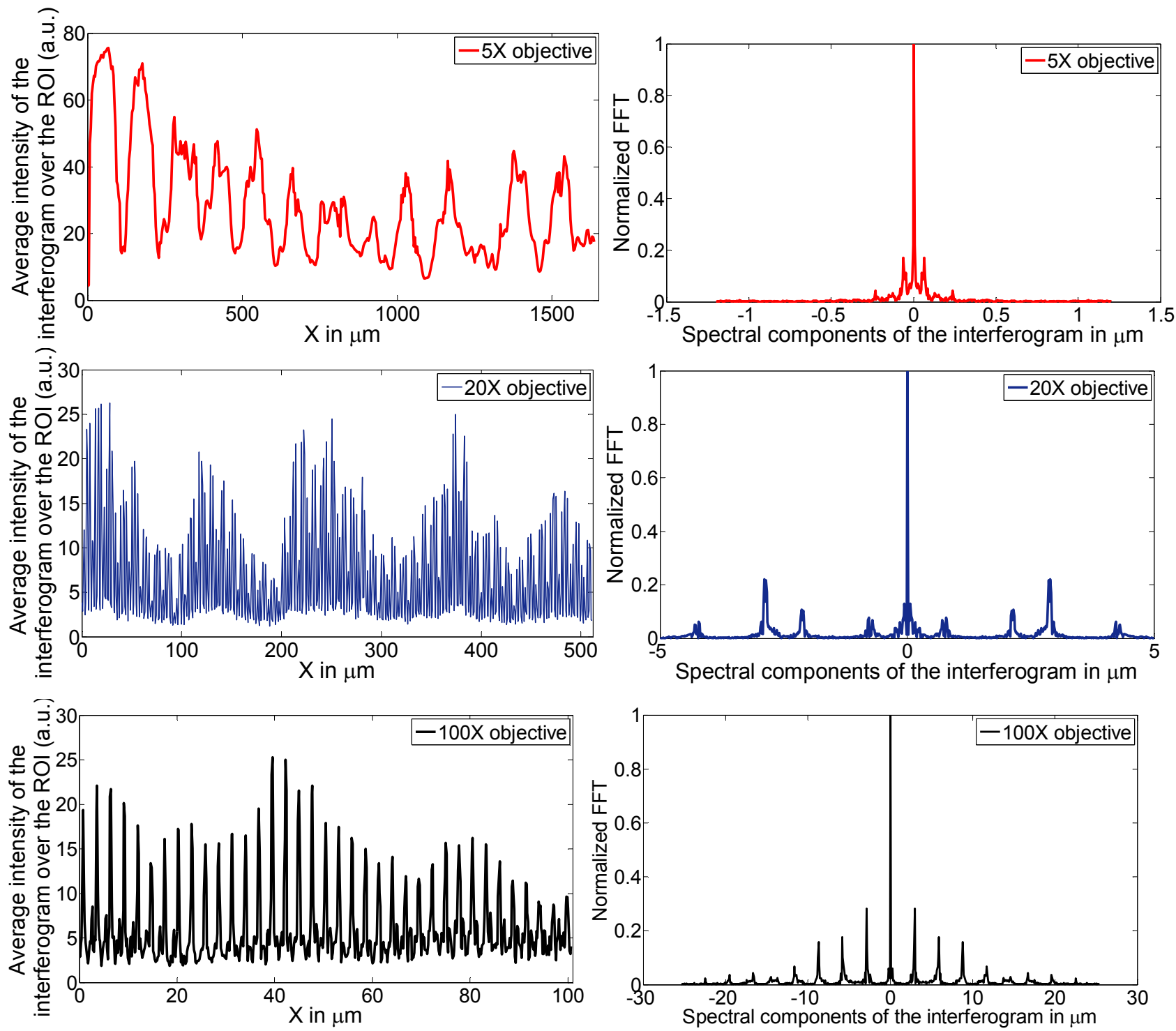

Figure 7. (Left) Average profile of the measured interferogram over the Region Of Interest (ROI) for microscopic objectives of 5X, 20X and 100X. (Right) Fourier Transform of the interferograms on the left.

Last but not least, we notice an apodization over the field of view in the spatial domain. This is mainly attributed to several defects that alter the signal such as the uniformity of the nanocouplers and the aberrations of the imaging optics.

\section{CONCLUSION AND PERSPECTIVES}

To conclude, a miniaturized standing wave spectrometer based on polymer waveguides has been presented. The fabrication process of the micro-nano structures has been detailed. A simple analytical model that predicts the spectral components content of the injected monochromatic light has been derived. Preliminary experimental results obtained with the $5 \mathrm{X}$ objective elucidate the peaks of the visible laser source. Future work will focus on the development of new designs for increased sampling and easier recovery of the measured spectra. 


\section{REFERENCES}

[1] Masters, B. R., "A Brief History of Spectral Analysis and Astrospectroscopy," Optics and Photonics News 20 (11), 34-39 (2009).

[2] http://www.britannica.com/EBchecked/topic/558901/spectroscopy/80590/Applications Article from the Encyclopedia Britannica,

[3] Bethe, H. A., and Salpeter, E. E., [Quantum Mechanics of One- and Two-electron Atoms], Academic Press, New York (1957)

[4] Demtröder, W., [Laser Spectroscopy: Basic concepts and instrumentation], Springer-Verlag, Berlin (1981)

[5] Stenholm, S., [Foundations of Laser Spectroscopy], Wiley \& Sons, California (1984)

[6] http://research.pbsci.ucsc.edu/chemistry/li/teaching/chem268/Spectroscopic\%20techniques.pdf

[7] Sprague, M. K., [Cavity ringdown spectroscopy, kinetics, and quantum chemistry of atmospherically relevant reactions], Dissertation (Ph.D.), California Institute of Technology (2012).

[8] Malak, M., Gaber, N., Marty, F., Pavy, N., Richalot, E., Bourouina, T., "Analysis of Fabry-Perot optical microcavities based on coating-free all-Silicon cylindrical Bragg reflectors," Optics Express 21(2), 2378-2392 (2013)

[9] Ferrari, A. C., and Basko D. M., "Raman spectroscopy as a versatile tool for studying the properties of graphene," Nature Nanotechnology 8, 235-246 (2013)

[10] El-Aneed, A., Cohen, A., Banoub, J.,"Mass Spectrometry, Review of the Basics: Electrospray, MALDI, and Commonly Used Mass Analyzers," Applied Spectroscopy Reviews 44, 210-230 (2009).

[11] Darbeau, R. W.,"Nuclear Magnetic Resonance (NMR) Spectroscopy: A Review and a Look at Its Use as a Probative Tool in Deamination Chemistry," Applied Spectroscopy Reviews 41, 401-425 (2006).

[12] Smith, B. C., "Fundamentals of Fourier Transform Infrared Spectroscopy," CRC press, Taylor \& Francis group, Florida (2011).

[13] Malak, M., Pavy, N., Marty, F., Peter, Y.-A., Liu, A. Q., Bourouina, T., "Micromachined Fabry-Perot resonator combining submillimeter cavity length and high quality factor," Applied Physics Letters 98 (21), 211113-1/3 (2011).

[14] Malak, M., Marty, F., Nouira, H., Vailleau, G., Bourouina, T., "All-silicon Michelson instrument on chip: Distance and surface profile measurement and prospects for visible light spectrometry," Applied Physics Letters 102 (14), 141102-1/4 (2013).

[15] B Saadany, T Bourouina, M Malak, M Kubota, Y Mita, D Khalil, "A Miniature Michelson Interferometer using Vertical Bragg Mirrors on SOI," IEEE/LEOS International Conference on Optical MEMS and Their Applications, 50-51 (2006).

[16] Cheben, P., Schmid, J. H., Delâge, A., Densmore, A., Janz, S., Lamontagne, B., Lapointe, J., Post, E., Waldron, P., and Xu, D.-X., "A high-resolution silicon-on-insulator arrayed waveguide grating microspectrometer with sub-micrometer aperture waveguides,"Optics Express 15(5), 2299-2306 (2007).

[17] le Coarer, E., Blaize, S., Benech, P., Stefanon, I., Morand, A., Lérondel, G., Leblond, G., Kern, P., Fedeli, J. M., and Royer, P., "Wavelength-scale stationary-wave integrated Fourier-transform spectrometry," Nature Photonics, 1, 473-478 (2007).

[18]B. Guldimann and S. Kraft, "Focal plane array spectrometer: miniaturization effort for space optical instruments," Proc. SPIE 7930, MOEMS and Miniaturized Systems X, 79300O-1/14 (2011).

[19] Malak, M., Philipoussis, I., Herzig, H. P., Scharf, T., "Polymer based single mode optical waveguide for spectroscopy applications", Proc. SPIE 8846, Optical Engineering + Applications, 884615-884615-7 (2013). 